\title{
Smart Powered Wheelchair Platform Design and control for People with Severe Disabilities
}

\author{
Youcef Touati ${ }^{*}$, Arab Ali-Cherif \\ Computer Science \& Artificial Intelligence Lab. LIASD, University of Paris 8, Saint-Denis, 93526, France
}

\begin{abstract}
Develop ment of new systems for disabled and elderly people's assistance requires a multidiscip linary approach based on new technologies according to users' needs. Intelligent wheelchairs can help this category of people to live more independently. The purpose of this paper is to propose and develop embedded control architecture for smart wheelchair monitoring, leading to gain mobility and independency. The control architecture uses virtual impedance principle and is based on some considerations concerning external force-feedback joystick abilities to translate variations of distances between the wheelchair and obstacles into a perceived effort. To test the effectiveness and evaluate performances of the proposed control architecture, some simulations and experimentations on a smart wheelchair called LIASD-WheelChair, are designed in this respect.
\end{abstract}

Keywords Smart Wheelchairs, Virtual Impedance Control, W ireless Commun ication, HMI

\section{Introduction}

Development of new systems for disabled and elderly people's assistance requires a multidisciplinary approach based on new technologies according to the users' needs. Researches in medical, paramedical and advanced technologies fields can collaborate directly to develop new strategies ranging from simple assistance to various access services such as websites, Smart phone and so on. As a kind of rehabilitation systems, intelligent wheelchairs can play an important role in helping the handicapped and the elderly people to live more independently and autonomously[1-2].

One of the main trends in s mart wheelchairs development is how to ensure a reliable remote tele-operation task with obstacle avoidance in a constrained environment taking into account system's interactive behavior. Various methods have been investigated in this context such as edge detection[3], potential field[4] and impedance control[5]. In edge detection methods, the objective is to determine the vertical edges of the obstacle and consequently attempts to steer the mobile system around an edge. The main drawback is the proximity between the mobile systemand the obstacles which is not suitable for the real time computation. Potential field methods allow a collision avoidance motion planning by generating a virtual force against obstacles and toward the goal. An attractive force between the mobile system and the target, and a repulsive force against obstacles are generated.

* Corresponding author:

touati@ai.univ-paris8.fr (Touati Youcef)

Published online at http://journal.sapub.org/ajss

Copyright (C) 2012 Scientific \& Academic Publishing. All Rights Reserved
As it's mentioned in[1], all these methods have advantage of making the fast motion planning for nearby obstacles, but with a shortcoming of getting into a local minimum where the attractive and repulsive forces are equal.

To overcome the local minimum, an extended virtual force field method is proposed, where a free vector is added to the repulsive force. This force becomes larger for the larger obstacles since repulsive forces are coming from all obstacle-detecting sensors, which makes this method unsuitable for certain applications. Impedance methods which are based on the concept of active control constitute a considerable contribution to deal with issues cited above. Both free and constrained motions can be handled by controlling the relation between input commands and robot-environment contact forces. In the same manner, impedance methods based on virtual aspects [6] are used to determine a repulsive force for mobile robot navigation avoiding obstacles. The principle is to generate a virtual force between mobile system and environment using exteroceptive localization tools such as US sensors, LIDAR and so on. In the most cases, these generated virtual forces are transferred and translated to a human operator through a joystick as tactile information. In this paper, we are interested by this kind of approach to monitor a smart wheelchair through a wireless communication network based 802.11 standards.

The remainder of this paper is organized as follow: in section 2, we present some related works concerning smart wheelchairs research projects. Section 3 describes experimental setup overview called LIASD-WheelChair where hardware and software aspects are illustrated. In order to ensure a reliable remote tele-operation task in a 
constrained environment, in section 4, we've proposed a strategy for wheelchair monitoring and control based on virtual impedance concepts. Experimental and simulation results are outlined in section 5. Finally, a brief conclusion and perspectives are summarized in the last section.

\section{Some Related Works}

Several research projects[7-10] using novel emerging technologies have been directed in the last decade to conceive and develop new hardware and software architectures to enhance the quality of service in terms of autonomy, mobility and accessibility. As an example, a TAO-2 project has been developed to increase the efficiency use of smart wheelchair[11] by establishing a methodology to design, implement, and test an effective add-on autonomy management system for use in conjunction with most common commercially available power wheelchairs. An adaptive, flexible and intelligent assistive technology has been proposed enabling users to participate fully in their daily lives[12-13]. An anti-collision and navigation systems aims to provide safe and intuitive means of mobility for people who have cognitive disabilities. In[14], intelligent ass is tance-sys tem has been conceived to facilitate driv ing for severe disabled and elder people with heavy reduced physical and/or mental abilities. The idea is to make use of a cognitive model of the user to reduce considerably the amount of necessary user commands in order to enhance the dependability of the wheelchair control. A UT-Intelligent Wheelchair is a project that develops the notion of graceful motion for a robotic wheelchair motion[15]. T inMan[16] and Wheelesley[17] are two research projects that have been highlighted by designing control systems based on a graphical user interface that has success fully been integrated with an eye tracking system and single switch scanning as input methods. More recently, in[18], a Smart wheelchair uses a virtual interface displayed by an on-board projection system to implement a shared control framework that enables the user to interact with the wheelchair while it is performing an autonomous task. In[19], a modular smart wheelchair co mponent system SWCS which can be added to a variety of commercial power wheelchairs has been elaborated. It is designed for all traditional input methods accommodations and for compatibility with multiple brands of wheelchairs. A s mart wheelchair integrating intelligent controllers that helps people with physical disabilities overcome daily problems has been proposed in[20]. It's equipped with onboard processing and multiple sensors. The system operates as a completely autonomous mobile robot platform. An intelligent control system allows giving more assistance to the users according to various sensors information such as cameras, laser range finder, and an onboard processing. In[21], a collaborative wheelchair assistant WCA is proposed for a full use of human skills by involving the us er into the navigation control. The user gives the high-level commands and directly controls the speed, while the low-level control is taken over by the machine, which is tracking a software defined guide path. Based on some successful experiments results, path guidance brings safe motion and drastically simplifies the control. Hence, the wheelchair user adopts an optimal driving behavior fro $m$ the first trial with a minimum of intervention. In the same manner, in[22], a research derived from the field of mobile robotics has been investigated to show how collaborative control techniques can be used to insure a best user-powered wheelchair interaction, predicting user intentions and responding to these predictions with adaptable levels of assistance. The same studies as in[23], illustrate how collaborative controls between user and wheelchair can be benefit. Another project allowing severely disabled people suffering from paraplegia and quadriplegia to steer an automated wheelchair is proposed[24]. It consists on a combination of a non-invasive EEG-based human-robot interface and an autonomous navigation system that safely executes issued commands. In[25], a s mart and automated wheelchair is experimented in complex navigational situations. For a given task, a conventional joystick and a proportional head-joystick can be used to assist the operator in his manoeuvring. Altering the translational and rotational velocities in situations where an obstacle blocks the user-commanded way, the driving assistance module significantly improves driver-performance by preventing all collisions along the way.

The work presented in this paper has the same perspectives as those concerning research projects mentioned above. It investigates one of the main trends in smart wheelchairs development area, which is about how to ensure a reliable remote tele-operation task with obstacle avoidance in free and constrained environments taking into account system' interactive behavior. Thus, Impedance control method based on the concept of active control can be considered also as an important contribution to deal with issues cited above. It provides a unified approach to all aspects of manipulation. Both free motion and contact tasks can be controlled using a single control algorithm. This method implements an algorithm that controls the relation between a velocity command and the contact force, which models the interconnection between an uncertain environment and a robot as impedance. In virtual impedance method, impedance is used to determine a repulsive force for mobile robot navigation avoiding obstacles. As an example, the principle of this method is used in[26], where the objective is to generate a virtual force between a wheelchair and the environment, which, in addition to the visual information, is transferred to a human operator through a joystick as tactile information.

In this paper, we are interested by this kind of control and particularly those concerning virtual impedance methods. For this purpose, we've proposed and developed embedded control architecture for smart wheelchair control and monitoring. The approach uses virtual impedance concept and is tested on experimental platform called LIASD-WheelChair. The implementation is based on some 
considerations concerning external force-feedback joystick abilities to translate variations of distances between a smart wheelchair and obstacles into a perceived effort. The approach exploits also information provided by Wireless Internet Camera Server, which is mounted on the wheelchair headrest, enabling the user to refine the odometry by computing the distance of objects from the wheelchair and then determine the safest routes to the desired location.

In what follows, we present the experimental platform called LIASD-WheelChair, developed in our laboratory in collaboration with Engineering Research Center CRIIP of Orsay University.

\section{Experimental Setup Overview}

\subsection{Embedde d Architecture}

LIASD-Wheelchair prototype is an adjustable adults' powered wheelchair (Figure.1). It has the same mechanical structure as Storm3 and can be monitored according to two operating modes: local mode and remote mode via wireless communication network based on 802.11 standards. It is suitable for indoor or outdoor use, has a range of $30 \mathrm{~km}$, and has the capacity to climb $35 \%$ slopes and $15 \mathrm{~cm}$ kerbs. The wheelchair includes some standard features: a non-powered seating platform, adjustable removable armrests with embedded joystick, adjustable swinging leg-rests and a head-rest incorporating an embedded camera. The wheelchair is fitted with four ultrasonic (US) sensors to identify obstacles from distance of $3 \mathrm{~cm}$ to $6 \mathrm{~m}$, with 3 to $4 \mathrm{~cm}$ of resolution. Our system includes two optical incremental encoders with resolution of 500 Counts per Revolution.

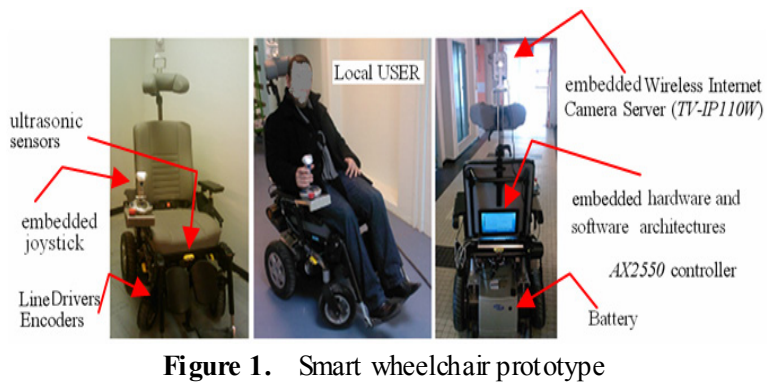

Figure. 2 shows different modules interconnection (remote computer, embedded laptop, different sensors, .etc.) for a better data flow using both wired and wire less networks.

The wireless network is based on IEEE 802.11 standard. The wheelchair can be controlled remotely using external force-feedback joystick. An embedded laptop mounted at the rear of the wheelchair allows managing US sensors via Fiveco card and controls Roboteq card using respectively an Ethernet and serial RS232 links.

In order to ensure navigation and anti-collision objectives a Wireless Internet Camera Server (TV-IP110W) is mounted on the wheelchair headrest. Images from the camera can be used to refine the odometry by computing the distance of objects from the wheelchair and then determine the safest routes to the desired location taking into account collision avoidance strategy. The wheelchair embeds also a USB joystick which is a little bit more sophisticated than traditional one. The joystick which is a SpeedLink SL-6612 has many configurable buttons that makes the navigation easier.

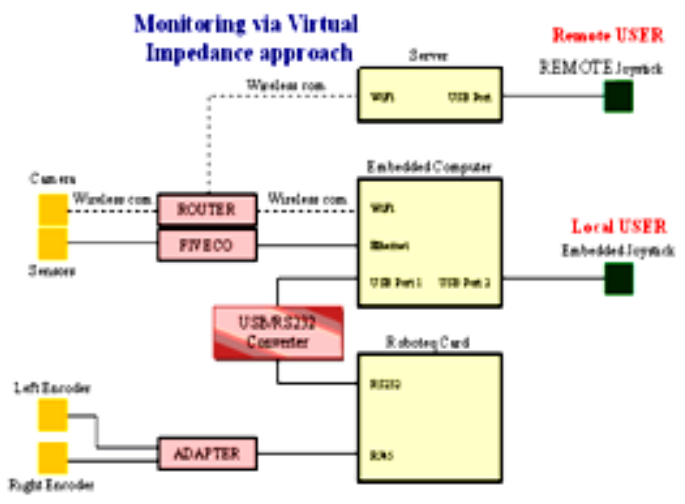

Figure 2. Communication scheme

The embedded control architecture is structured into three levels: basic control level, tactical level and strategy level (Figure.3)

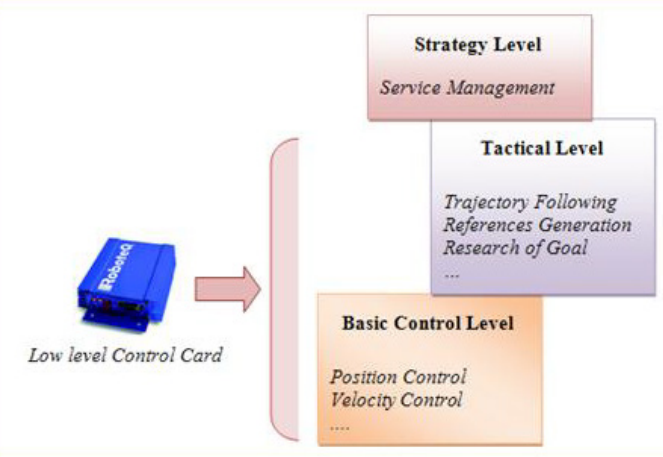

Figure 3. Control architecture

The strategy level defines the way the wheelchair can achieve the main goal previously defined. Algorithms such as planning trajectories, localization, etc. are implemented to fulfill and ensure a desired task. Thus, a set of elementary actions are generated in the tactical level aiming to satisfy reached goals. In the basic control level, a PID control using an AX2550 controller is implemented. It can be configured in a speed or position mode using a selection matrix.

\subsection{Communication Archi tecture}

Figure. 4 illustrates the global communication and control architecture of the platform.

An I2C protocol is used to manage US sensors module via a Fiveco card, and implement USB and RS232 communicat ion bus for embedded laptop (Asus-EeePC-10002H) and AX2550 controller interconnection. Via an adapter, incremental encoders are connected to Roboteq card trough RJ45 link.

An embedded Asus-EeePC-1002H laptop use to control the whole architecture of the wheelchair, includes some 
features allowing a better information handling of different embedded modules (FiveCo, RoboTek, TRENDnet-TV camera,...) as well as those remotely located (server, routers ,...) via a wire less network.

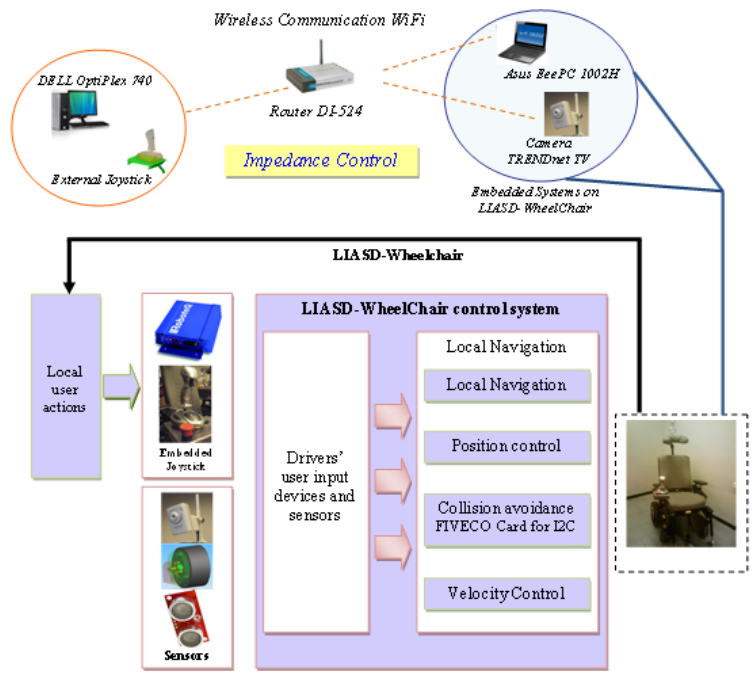

Figure 4. Control and communicat ion net work scheme

Based on wireless IEEE 802.11 standard network, the wheelchair can be controlled and monitored continuously using an external joystick. In order to refine the odometry, information derived from wireless internet camera server allow the user to estimate distances between objects and the wheelchair thus to determine the safest routes to the desired location by avoiding collisions. This kind of monitoring can find its usefulness in some constraining situations as binding postures of the wheelchair in the environment, unconscious ness or tiredness of disabled people, and so on.

In this paper, we are interested by this kind of communication network. The objective is to ensure a reliable remote tele-operation task in a constrained environment by proposing a strategy for LIASD-WheelChair monitoring and control based on virtual impedance concepts. The control strategy is presented in the next section.

\section{Virtual Impedance Approach}

\subsection{Proposed Approach Principles}

Since the works of Hogan in[27], impedance control approach is considered as one of the most frameworks to control the interactions between system and environment. This kind of approach ensures a smooth implementation of desired tasks by taking into account some real impedance parameters properties such as inertia, $v$ iscosity and stiffness, so that:

$$
F_{e}=M_{e} \cdot d \ddot{X}_{e}+B_{e} \cdot d \dot{X}_{e}+K_{e} \cdot d X_{e} .
$$

Where $F_{\mathrm{e}}$ describes the interaction force between system and environment and $M_{\mathrm{e}}, B_{\mathrm{e}}$ and $K_{\mathrm{e}}$ represent respectively the desired inertia, $v$ iscosity and stiffness of the considered system.
The displacement between the current position of the system $X_{\mathrm{e}}$ and the desired one $X_{\mathrm{d}}$, can be defined as follows:

$$
d X=X_{e}-X_{d} .
$$

The principle of classical impedance is clearly illustrated bellow (Figure.5). Since no effort is exerted until making contact between system and environment, this approach is not useful[16]. Recent researches have been directed towards the development of new concepts based on VI principles using visual information[17][18].

Based on these works, and particularly on those developed in[16], we propose in this paper a non-contact impedance control approach for smart wheelchair monitoring through wireless communication based on 802.11 standard.

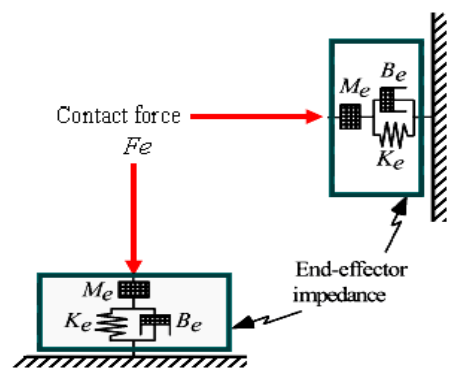

Figure 5. Impedance interpretation.

As shown on Figure.6, virtual impedance is represented as spherical configuration between a mobile system and environment obstacles. Thus, when the mobile comes inside the virtual sphere, a virtual force is then generated before any contact.

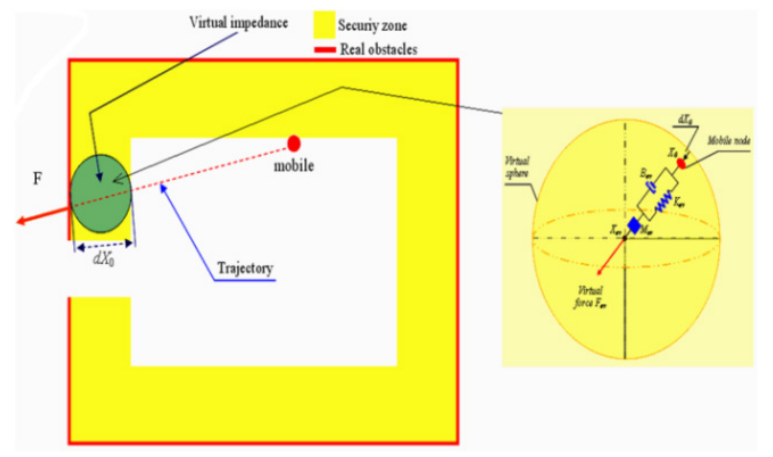

Figure 6. Virtual impedance representation

Let us consider now, the case when the mobile approaches a security zone, and set a virtual sphere with radius $r$ at the center of the mobile.

When the mobile comes into the interior of the virtual sphere, the normal vector from the surface of the sphere to the mobile $d X_{0}$ can be written as:

$$
d X_{0}=X_{r}-r \cdot n \text {. }
$$

Where $X_{r}=X_{0}-X_{e v}$ represents the displacement vector of the mobile from position $X_{0}$ to the center of the sphere $X_{e v}$.

The vector $n \in \mathfrak{R}^{l}$ is given by:

$$
n=\left\{\begin{array}{ll}
\frac{X_{r}}{\left|X_{r}\right|} & \text { si }\left(\left|X_{r}\right| \neq 0\right) \\
0 & \text { si }\left(\left|X_{r}\right|=0\right)
\end{array}\right. \text {. }
$$


If the mobile node moves inside the virtual sphere $\left(\left|X_{r}\right|<r\right)$, virtual impedance can be highlighted by computing a virtual force-feedback $F_{e v} \in \mathfrak{R}^{l}$ exerted on the center of that sphere as follows:

$$
F_{e v}=\left\{\begin{array}{lll}
M_{e v} \cdot d \ddot{X}_{0}+B_{e v} \cdot d \dot{X}_{0}+K_{e v} \cdot d X_{0} & \text { si } & \left(\left|X_{r}\right|<r\right) \\
0 & \text { si } & \left(\left|X_{r}\right| \geq r\right)
\end{array}\right.
$$

Where, $M_{e v}, B_{e v}$ and $K_{e v}$ represent respectively the virtual inertia, viscosity and stiffness. One can note that the virtual force $F_{e v}$ becomes zero when the mobile is not in the virtual sphere or is at the center of that sphere.

Moreover, one can note that a closed loop speed mode is performed using a full featured PID algorith $\mathrm{m}$. The ultimate goal in well tuned PID parameters is to allow reaching the desired speed quickly without overshoot or oscillation. In that way, every 16 milliseconds, the controller can measure the actual motor speed and subtracts it from the desired position to compute the speed error. The resulting error value is then multiplied by a user selectable Proportional Gain. The effect of this part of the algorithm is to apply power to the motor which is proportional with the difference between the current and desired speed: when far apart, high power is applied, with the power being g radually reduced as the motor moves to the desired speed.

\subsection{Software As pects}

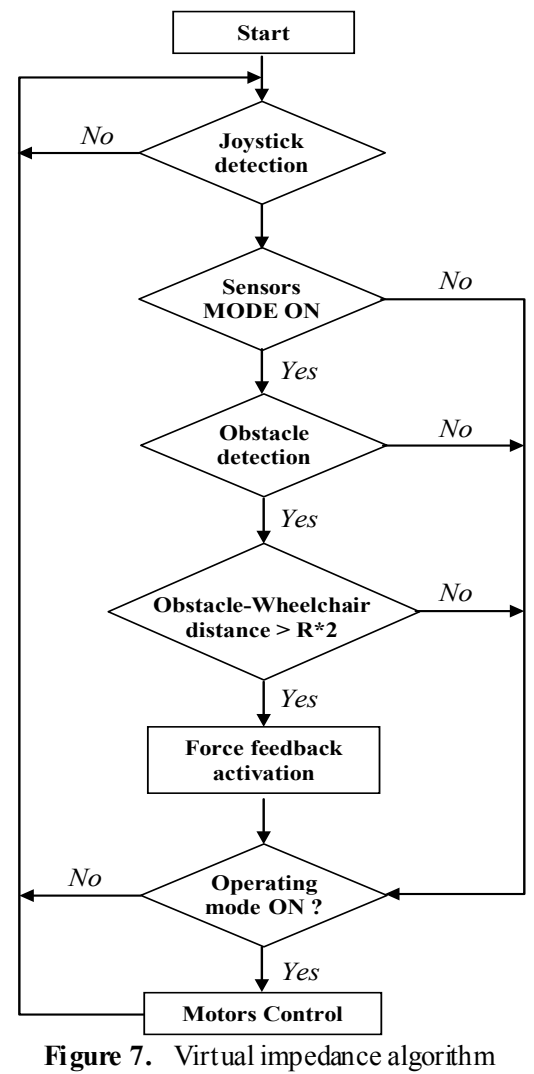

This approach is implemented according to some considerations concerning force-feedback joystick abilities to monitor remotely LIASD-WheelChair taking into account environment obstacles (Figure.7). The proposed algorithm for our application shows that according to US sensors data, the perceived efforts as tactile information on the joystick allow the users to take the right decisions for wheelchair monitoring.

As shown on Figure.8, we have developed a software architecture including a set of classes taking into account the application entirely. Thus, G_SDL_Jostick and joy_imp1 represent two classes developed to handle the force-feedback joystick module allowing the user to monitor remotely the wheelchair.

We note that these classes are developed with Qt and implemented under directInput tool.

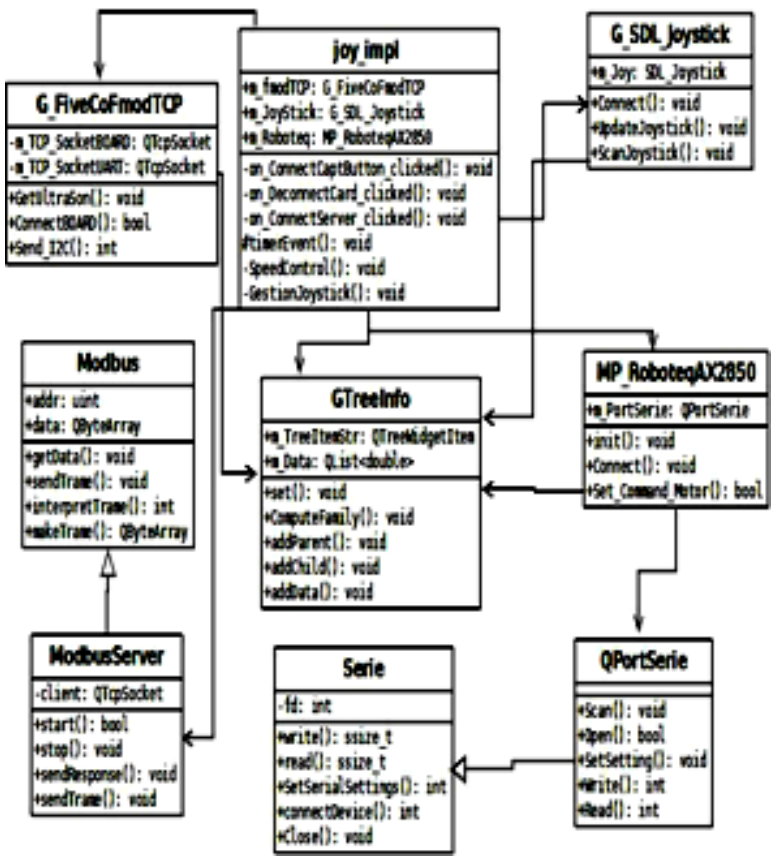

Figure 8. Soft ware architecture

In order to show the contribution of virtual impedance approach, we have realized a set of simulations and experimentations.

\section{Simulation and Experimental Results}

The objective is to perform some tests in order to analyse the behavior of the LIASD-WheelChair interacting with environment. Thus, based on generated virtual forces that are transferred and translated through a joystick as tactile information, the human operator can perform a set of movements to maneuver the wheelchair. In this context, as a model of environment, we have proposed a circular configuration with a real behavior model of LIASD-WheelChair. One can note that different models of environment can be chosen such as square, ellipse, etc.

First, virtual impedance para meters $K_{e v}, B_{e v}$ and $M_{e v}$ are set arbitrarily and respectively to the following values $50[\mathrm{~N} / \mathrm{m}]$, $10[\mathrm{Ns} / \mathrm{m}]$ and $2[\mathrm{~kg}]$. Notice that the stiffness of the object is much $h$ igher that the positional stiffness of the impedance, so 
that the environment can be considered rigid. Moreover, for Sample-axis, each second corresponds to 100 Samples.

Figure.9, illustrates the exerted efforts by the joystick in the environment (Environment 1). Indeed, from samp le 250, we note an increasing of the perceived efforts on the joystick corresponding to the first contact between the wheelchair and the virtual sphere. At this time, the reached value is 1100 and oscillates around 1000 and 1250 . After sample 2000, the perceived force decreases to a minimum value of 400 . Despite this, the intensity remains very weak.
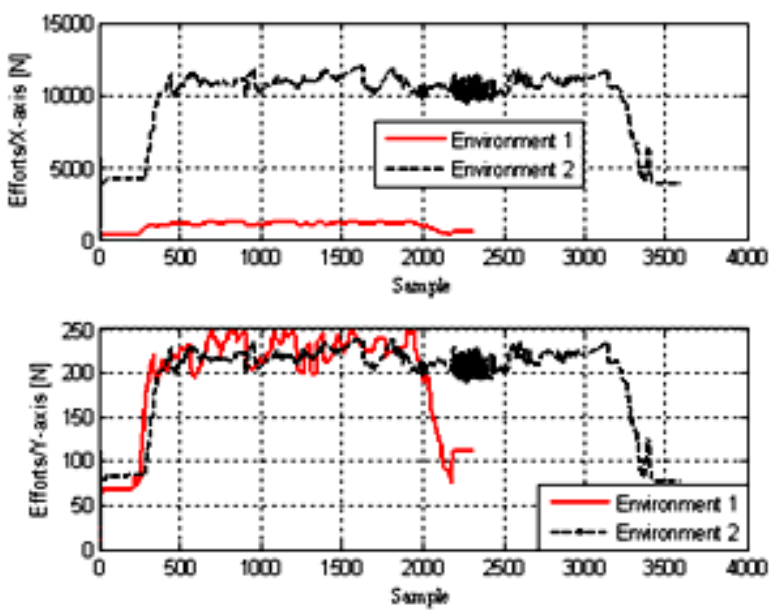

Figure 9. Exerted efforts on the environment

From Figure.10, one can see that the real trajectory of wheelchair (Environment 1) doesn't reflect the desired one, but it also goes beyond the specified environment. This can be explained by the fact that the virtual stiffness has a small value which leads to a weak force felt on the joystick.

To show the interest of the proposed virtual impedance method, we have simulated another implementation with different value of $K_{e v}$. Thus, we have integrated a value of $250[\mathrm{~N} / \mathrm{m}]$. Thus, as we can see fro $\mathrm{m}$ Figure. 9 and Figure.10, the joystick set a real trajectory which doesn't go through the desired one (Environment 2). The fact that the stiffness value is significant, the perceived efforts are also significant (Environment 2) leading thus, to make the wheelchair follow the trajectory without any direct contact with real environment.

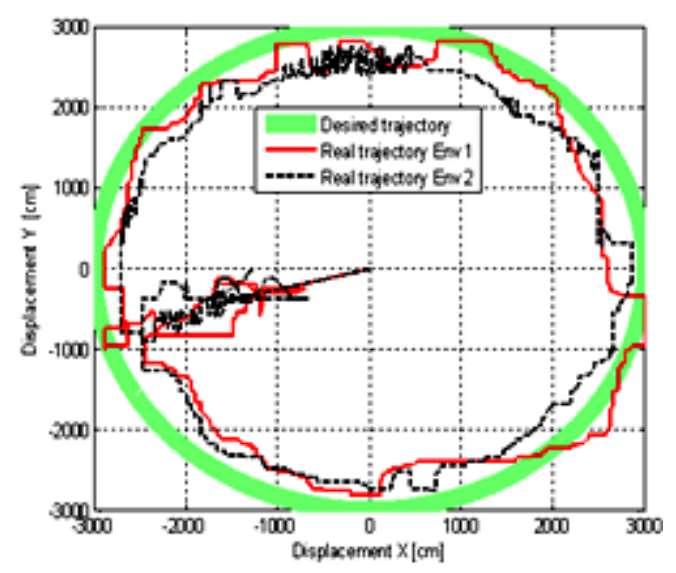

Figure 10. Desired and real trajectories
These results show that the increasing of the perceived efforts, from sample 300 , to the value of 1000 corresponds to an elementary displacement $d X_{0}$ of $20[\mathrm{~cm}]$. Th is illustrates the first contact between the wheelchair and the virtual sphere. These efforts tend to oscillate respectively between 1200 and 25 accord ing to $\mathrm{X}$ and $\mathrm{Y}$-axis.

Figure. 11 and Figure. 12 show respectively the wheelchair trajectories according to $\mathrm{X}$-axis and $\mathrm{Y}$-axis for different values of environment stiffness $K_{e v}=50[\mathrm{~N} / \mathrm{m}]$ and $250[\mathrm{~N} / \mathrm{m}]$. According to changing parameters like those of environment and robot-environment interaction, the controller must be adaptive to time-variable system, which is an important feature for its implementation. In fact, when the wheelchair motion is constrained by the environment, its trajectory is modified using contact forces. This can be considered as a disturbance which could rapidly change the global behaviour of the task. In this case, the use of virtual impedance control is very efficient and the corresponding results are very close to the desired ones, except some peaks indicating virtual contacts between the wheelchair and the environment. When contact forces between parts arise, along a direction, the corresponding results exhibit a peaks then decreases and oscillate around the desired values.

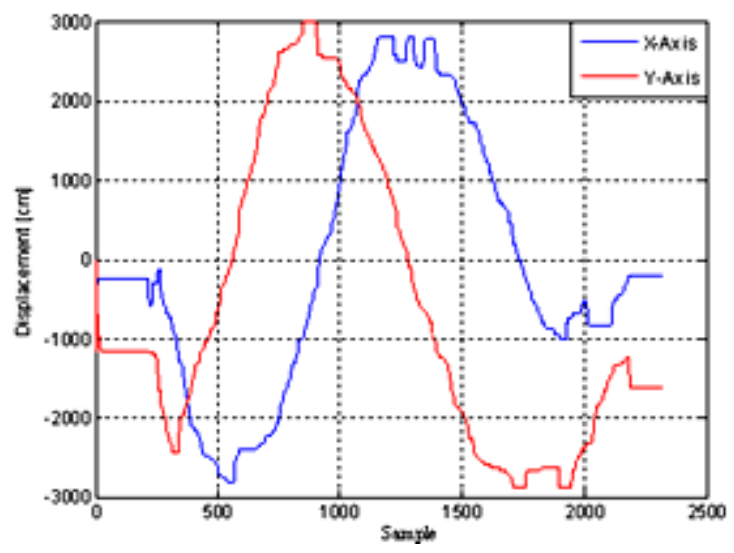

Figure 11. Realtrajectories for $\mathrm{Kev}=50[\mathrm{~N} / \mathrm{m}]$

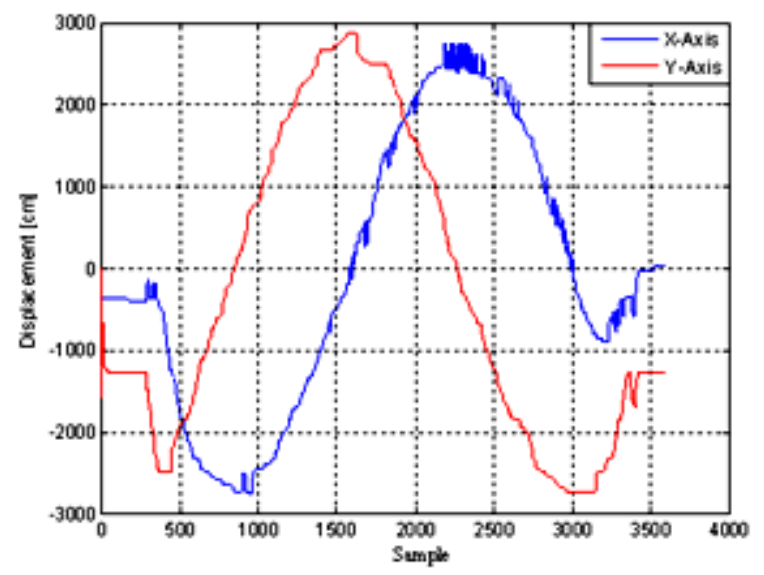

Figure 12. Realtrajectories for $\mathrm{Kev}=250[\mathrm{~N} / \mathrm{m}]$

To illustrate the usefulness of the proposed virtual impedance approach for LIASD-WheelChair remote monitoring experimentally, we have performed some tests. 
For this purpose, information coming from various sources, i.e., US sensors and wireless camera, through wireless network allow the human operator to ensure a right positioning a sweet manoeuvrability of the wheelchair.

Thus, Figure.13 illustrates different postures of the wheelchair. Here, the monitoring is ensured using both an external and embedded joysticks with identical characteristics. Thus, to ensure a safe control of the wheelchair according to some tele-operated movements of the joysticks, a specific PID parameters related to speed controller have been determined, so that, Proportional, Differential and Integral components values are respectively $K_{p}=2, K_{d}=2$ and $K_{i}=2$.

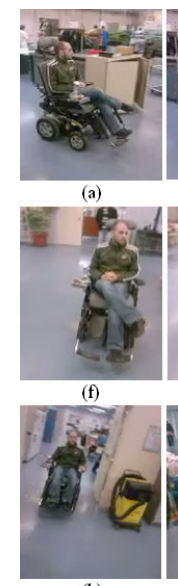

(k)
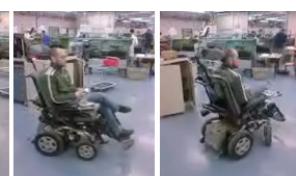

(b)

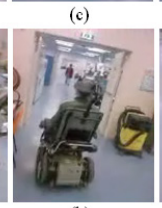

(g)

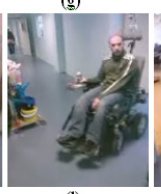

(I)

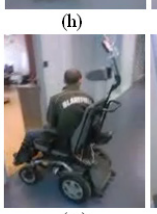

(m)
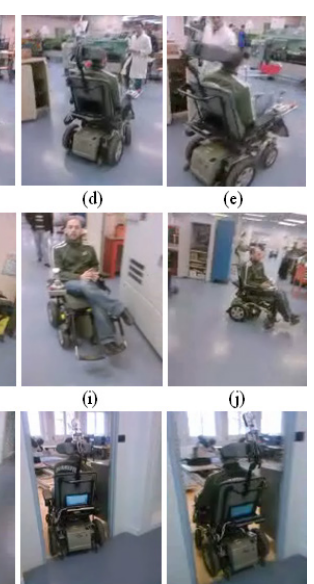

(n)

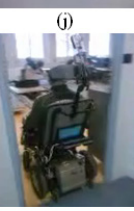

Figure 13. LIASD-WheelChair moving in constrained environment

Using Wi-Fi communication mode (Figure. 13 (a)-(j)), the wheelchair performs a good trajectory in constrained environment. In this case, an external joystick is requested to ensure the wheelchair mon itoring continuously. This kind of control transition can happen in emergency cas es particularly, when the whee lchair doesn't respond to some desired control actions. Moreover, the use of a Wireless Internet Camera Server, which is mounted on the wheelchair headrest, enables the user to refine the odometry by computing the distance of objects from the wheelchair and then determine the safest routes to the desired location by avoiding collisions. In Figure.13 (k)-(m), the wheelchair is locally monitored by the user. Here, as in tele-operated mode, via a force feedback joystick the user perceives virtual forces leading him to have a better monitoring.

\section{Conclusions}

In this paper we have proposed an approach dedicated for improving handicapped people's assistance and particularly those concerning smart wheelchairs. We have presented an approach based on virtual impedance method leading to control and to monitor LIASD-Wheelchair remotely through wireless communication network which is based on 802.11 standards. This kind of approach allows a reliable remote manoeuvring with obstacle avoidance in a constrained environment taking into account system's interactive behavior. In order to test the effectiveness of the proposed approach, simulations and experimentations have been designed in this respect. Thus, according to the obtained results, some future researches and perspectives involving new techniques such as neural networks and fuzzy logic should be undertaken in order to increase performances in terms of acces sibility and autonomy. These pro mis ing results lead us to further investigation into the use of this control approach for other kinds of tasks

Some other research perspectives can be investigated in the same direction as projects mentioned above, contributing to propose new software and hardware architectures based on emerg ing technologies such as wireless sensor network, RFID and web-services in order to improve autonomy, mobility and accessibility of disabled people in their daily live.

\section{ACKNOWLEDGEMENTS}

Authors would like to thank the Computer Science Department of Paris-8 University for its financial support and the Engineering Research Center CRIIP team of ENS Cachan for their availability and technical collaboration.

\section{REFERENCES}

[1] Tao Lu, Kui Yuan, Haibing Zhu and Huosheng Hu, "An embedded control system for intelligent wheelchair", in Proceedings of 27th Annual International Conference of the IEEE En gineering in Medicine \& Biology Society, Shanghai, China, 2005.

[2] T. Rofer and A. Lankenau, , "Ensuring safe obstacle avoidance in a shared-control system", in Proceedings of 7th IEEE International Conference on Emerging Technologies and Factory Automation, vol.2, pp.1405-1414, July 1999.

[3] J. Borenstein and Y. Koren, "Obstacle avoidance with ultrasonic sensors", IEEE Journal of Robotics and Automation, vol.RA-4, no.2, pp.213-218, 1988.

[4] J. Borenstein and Y. Koren, "Real-time obstacle avoidance for fact mobile robots", IEEE Transaction on System, Man and Cybernetics, vol.19, no.5, pp.1179-1187, 1989.

[5] N. Hogan, "Impedance control: An approach to manipulation", Part I, Part II, Part III, ASME J. Dynamic Systems, Measurements and Control, vol.107, no.1, pp.1-24, 1985.

[6] J. Ota, T. Arai, E. Yoshida, D. Kurabayashi and T. Mori, "Real time planning method for multiple mobile robots", in Proceedings of the IEEE International Symposium on Assembly and Task Planning, pp.406-411, 1995.

[7] R.C. Simpson, "Smart wheelchairs: A literature review", Journal of Rehabilitation Research \& Development, vol.42, no.4, pp. 423-436, 2005.

[8] C. Gao, I. Hoffman, T. Panzarella and J. Spletzer, "ATRS - A Technology-based solution to automobility for wheelchair 
users", in Proceedings of 6th International Conference on Field and Service Robotics, vol. 42, 2007.

[9] M. Maso and J.C. Garcia et al, "Experiences in assisted mobility: the SIAMO project", in Proceedings of the IEEE International Conference on Control Applications, vol.2, pp. 766-771, Sept 2002.

[10] R.A.M. Braga, M. Petry, A.P. Moreira and L.P. Reis, "INTELLWHEELS: A development platform for intelligent wheelchairs for disabled people", ICINCO 2008, International Conference on Informatics in Control, Automation and Robotics, Funchal Madeira (Portugal), pp.115-121, May 2008.

[11] G. Takashi, "The TAO Project: Intelligent wheelchairs for the handicapped", AAAI Technical Report FS-96-05, pp.28-37, 1996.

[12] A. Mihailidis, P. Elinas, J. Boger and J. Hoey, "An intelligent powered wheelchair to enable mobility of cognitively impaired older adults: An anti-collision System", IEEE Transactions on Neural Systems \& Rehabilitation Engineering, 15(1), pp.136-14, 2007.

[13] P. Viswanathan, J. Boger, J. Hoey, P. Elinas and A. Mihailid is, "The future of wheelchairs: Intelligent collision avoidance and navigation assistance", Geriatrics and Aging Magazine, 10(4), pp.253-256, 2007.

[14] M. Jipp, A. Wagner and F. Badredine, "Individual ability-based sy stem design of dependable human-technology interaction", the 17th IFAC World Congress, Seoul, South Korea, July 2008.

[15] S. Gulati and B. Kuipers, "High performance control for graceful motion of an intelligent wheelchair", IEEE International Conference on Robotics and Automation, Pasadena, California, May 2008.

[16] D. Miller and M. Slack, "Design and testing of a low-cost robotic wheelchair prototype", Autonomous Robots, vol.1, no.3, 1995.

[17] H. Yanco, "Wheelesley, a robotic wheelchair system: indoor navigation and user interface", Lecture Notes in Artificial Intelligence: Assistive Technology and Artificial Intelligence, pp.256-268, 1998 .

[18] S.P. Parikh, V. Grassi, V. Kumar, and J. Okamoto, "Incorporating user inputs in motion planning for a smart wheelchair", in Proceedings of the IEEE International
Conference on Robotics and Automation (ICRA), New Orleans, LA, pp.2043-2048, 2004.

[19] R.C. Simpson, "Smart Wheelchair. A literature review", Journal of Rehabilitation Research and Development, JRRD, 42(4), pp.423-436, 2005.

[20] S. Parikh, V. Grassi and V. Kumar, "Integrating human inputs with autonomous behaviors on an intelligent wheelchair platform". IEEE Intelligent Systems: Special Issue on Interacting with Autonomy, 22(2), pp.33-41, April 2007.

[21] Q. Z. Burdet, E. Rebsamen and B.C.L. Teo, "Evaluation of the collaborative wheelchair assistant System", IEEE 10th International Conference on Rehabilitation Robotics, ICORR'07, Noordwijk, Netherlands, pp.601-608, 2007.

[22] T. Carlson and Y. Demiris, "Human-wheelchair collaboration through prediction of intention and adaptive assistance", in Proceedings of IEEE International Conference on Robotics and Automation, ICRA'08, Pasadena, CA, pp.3926-3931, 2008.

[23] C. Mandel, T. Luth, T. Laue, T. Rofer, A. Graser and B. Krieg-Bruckner, "Navigating a smart wheelchair with a brain-computer interface interpreting steady-state visual evoked potentials", in Proceedings of IEEE/RSJ International Conference on Intelligent robots and systems, IROS'09, St. Louis, MO, 2009, pp.1118-1125, 2009.

[24] T. Carlson and Y. Demiris, "Increasing robotic wheelchair safety with collaborative control: Evidence from secondary task experiments", in Proceedings of the IEEE International Conference on Robotics and Automation, ICRA'10, Anchorage, Alaska, pp.5582-5587, 2010.

[25] T. Rofer, C. Mandel and T. Laue, "Controlling an automated wheelchair via joystick-head-joystick supported by smart driving assistance", IEEE International Conference on Rehabilitation Robotics, ICORR'09, Kyoto, Japan, pp.743-748, 2009.

[26] S.S. Lee and J.M. Lee, "Haptic interface design for the tele-surgery", in Proceedings of the CIDAM Workshop on Service Automation and Robotics, Hong Kong, pp.81-91, 2000 .

[27] N. Hogan, "Stable execution of contact tasks using impedance control", IEEE International Conference on Robotics and Automation, Raleigh, North Carolina, USA, pp.1047-1054, 1987. 